\title{
Kernel-based collocation methods for Heath-Jarrow-Morton models with Musiela parametrization
}

\author{
Yuki Kinoshita*1 and Yumiharu Nakano ${ }^{\dagger 1}$ \\ ${ }^{1}$ Department of Mathematical and Computing Science, \\ Tokyo Institute of Technology, \\ W8-28, 2-12-1, Ookayama, Meguro-ku, Tokyo 152-8550, Japan
}

September 8, 2020

\begin{abstract}
We propose kernel-based collocation methods for numerical solutions to HeathJarrow-Morton models with Musiela parametrization. The methods can be seen as the Euler-Maruyama approximation of some finite dimensional stochastic differential equations, and allow us to compute the derivative prices by the usual Monte Carlo methods. We derive a bound on the rate of convergence under some decay conditions on the interpolation functions and some regularity conditions on the volatility functionals.
\end{abstract}

Key words: Heath-Jarrow-Morton models, Musiela parametrization, kernel-based interpolation, collocation methods.

AMS MSC 2010: 65M70, 91G30, 60H15.

\section{Introduction}

In this paper, we are concerned with numerical methods for Heath-Jarrow-Morton (HJM) models with Musiela parametrization. Consider the forward rate process $f(t, T), 0 \leqslant$ $t \leqslant T<\infty$, given as a family of Itô processes, in an arbitrage-free bond market. Then, by Heath et.al [10], the process $f(t, T)$ should evolve according to

$$
d f(t, T)=\alpha(t, T) d t+\sum_{i=1}^{d} \sigma_{i}(t, T) d W_{i}(t) .
$$

Here, this equation is defined on a complete probability space $(\Omega, \mathcal{F}, \mathbb{P})$ with a filtration $\{\mathcal{F}(t)\}_{t \geqslant 0}$ satisfying the usual conditions. The probability measure $\mathbb{P}$ is interpreted as an equivalent local martingale measure as explained below. The process

*yk.lgb13@gmail.com

${ }^{\dagger}$ Corresponding author: nakano@c.titech.ac.jp 
$W(t)=\left(W_{1}(t), \ldots, W_{d}(t)\right), t \geqslant 0$, is a standard $d$-dimensional $\{\mathcal{F}(t)\}$-Brownian motion under $\mathbb{P}$. The processes $\sigma_{i}(t, T), i=1, \ldots, d$, are assumed to be appropriately measurable and integrable, and the process $\alpha(t, T)$ is given by

$$
\alpha(t, T)=\sum_{i=1}^{d} \sigma_{i}(t, T) \int_{t}^{T} \sigma_{i}(t, s) d s
$$

We refer to standard textbooks such as Musiela and Rutkowski [17], Shreve [22], Björk [3] and the references therein for details and developments of HJM models (1.1). Then, Musiela [16] shows that $r(t, x):=f(t, t+x)$, which is called the Musiela parametrization, is a mild solution to the stochastic partial differential equation

$$
d r(t, x)=\left(\frac{\partial}{\partial x} r(t, x)+\alpha(t, t+x)\right) d t+\sum_{i=1}^{d} \sigma_{i}(t, t+x) d W_{i}(t)
$$

in a suitable function space. The equation $(1.2)$ is called the Heath-Jarrow-MortonMusiela (HJMM) equation. Since then the existence and uniqueness of solutions to versions of (1.2) have been vastly studied. See, e.g., Goldys and Musiela [9], Filipović [8, Barski and Zabczyk [1, Kusuoka [15] and the references therein.

As for numerical methods for (1.2), Barth [2] studies the finite element methods and Dörsek and Teichmann [7] proposes a splitting up method. In the present paper, we examine kernel-based collocation methods for numerical solutions to 1.2 when $\sigma$ depends on $f(t, \cdot)$, whence on $r(t, \cdot)$, as an alternative to existing methods.

Given a points set $\Gamma=\left\{x_{1}, \ldots, x_{N}\right\}$ such that $0<x_{1}<\cdots<x_{N}$, and a positive definite function $\Phi: \mathbb{R} \rightarrow \mathbb{R}$, the function

$$
I(g)(x)=\sum_{j=1}^{N}\left(\left.K^{-1} g\right|_{\Gamma}\right)_{j} \Phi\left(x-x_{j}\right), \quad x \in \mathbb{R},
$$

interpolates $g$ on $\Gamma$. Here, $K=\left\{\Phi\left(x_{j}-x_{\ell}\right)\right\}_{j, \ell=1, \ldots, N},\left.g\right|_{\Gamma}$ is the column vector composed of $g\left(x_{j}\right), j=1, \ldots, N$, and $\left(\left.K^{-1} g\right|_{\Gamma}\right)_{j}$ denotes the $j$-th component of $\left.K^{-1} g\right|_{\Gamma} \in \mathbb{R}^{N}$. Since one can expect

$$
\frac{d^{m}}{d x^{m}} g(x) \approx \frac{d^{m}}{d x^{m}} I(g)(x), \quad m=0,1,
$$

replacing $r(t, \cdot)$ and $\partial r(t, x) / \partial x$ in the right-hand side in 1.2$)$ with $I(r(t))$ and $\partial I(r(t))(x) / \partial x$, respectively, gives a reasonable approximation of 1.2$)$, and the resulting equation leads to an $N$-dimensional stochastic differential equation collocated at the points in $\Gamma$. See Section 3 below for a more precise derivation. The methods using the kernel-based interpolation as in above are called kernel-based collocation methods in general, which are first proposed by Kansa [12] (see also Kansa [13, 14]). Since then many studies on numerical experiments and practical applications for the kernel-based methods are generated. Rigorous convergence issues are studied in, e.g., Schaback [21], Cialenco et.al [5], Hon et.al [11], Nakano [19, 20, 18]. Our aim is to address the kernel-based collocation methods in the problem of numerically solving HJMM equations and to obtain a bound 
on the rate of convergence for the methods. To this end, we use the stability result of the kernel-based interpolation with Wendland kernels proved in [20] and develop the error estimation result for the interpolation in a class of functions having relatively low regularities.

This paper is organized as follows. The next section is devoted to the proof of the existence and uniqueness result for $(1.2)$ in a Hilbert space that is suitable for our purpose. We describe the kernel-based collocation methods in details and derive the approximation error in Section 3 . In Section 4 we apply our numerical methods to the pricing problem of the caplets.

\section{HJMM equations}

We describe Heath-Jarrow-Morton models with Musiela parametrization or HJMM equations for interest rate modeling in a way suitable for our purpose. Our setup is based on 8, with a slight modification.

First, we introduce several notation. Let $\mathbb{R}_{+}=[0, \infty)$. For any open or closed set $V \subset \mathbb{R}$ we write $\mathcal{B}(V)$ for the Borel $\sigma$-field of $V$. We use Leb to denote the Lebesgue measure on $(\mathbb{R}, \mathcal{B}(\mathbb{R}))$. We put $L^{p}(V)=L^{p}(V, \mathcal{B}(V)$, Leb) for $p \in[1, \infty]$ and denote by $\|\cdot\|_{L^{p}(V)}$ its norm. We also denote by $L_{l o c}^{1}\left(\mathbb{R}_{+}\right)$the collection of all Borel measurable and locally Lebesgue integrable functions on $\mathbb{R}_{+}$. Denote by $C^{k}(V)$ the space of all $C^{k}$-functions on $V$, and by $C_{b}^{k}(V)$ the collection of all functions in $C^{k}(V)$ such that

$$
\|u\|_{C^{k}(V)}:=\sum_{m=0}^{k} \sup _{x \in V}\left|\frac{d^{m} u}{d x^{m}}(x)\right|<\infty .
$$

By $C$ we denote positive constants that may vary at each occurrence and that do not depend on time and spatial variables in $\mathbb{R}_{+}$, elements in $\Omega$ and $U$, and the approximation parameter $h$ introduced below.

We work in the Hilbert space

$$
U:=\left\{\begin{array}{l|l}
\phi \in L_{l o c}^{1}\left(\mathbb{R}_{+}\right) & \begin{array}{l}
\text { there exist the generalized derivatives } \\
\phi^{\prime}, \phi^{\prime \prime} \in L_{l o c}^{1}\left(\mathbb{R}_{+}\right) \text {of } \phi \text { such that }\|\phi\|_{U}<\infty
\end{array}
\end{array}\right\}
$$

with the norm $\|\cdot\|_{U}$ defined by

$$
\|\phi\|_{U}^{2}=|\phi(0)|^{2}+\left|\phi^{\prime}(0)\right|^{2}+\int_{0}^{\infty}\left(\left|\phi^{\prime}(x)\right|^{2}+\left|\phi^{\prime \prime}(x)\right|^{2}\right) w(x) d x,
$$

where $w: \mathbb{R}_{+} \rightarrow[1, \infty)$ is a nondecreasing $C^{1}$-function such that $w^{-1 / 3} \in L^{1}\left(\mathbb{R}_{+}\right)$.

We consider the mapping $S(t): U \rightarrow U$ defined by $S(t) \phi(x)=\phi(t+x), t, x \in \mathbb{R}_{+}$. It is clear that $\{S(t)\}_{t \in \mathbb{R}_{+}}$defines a semigroup on $U$. Moreover we have the following:

Proposition 2.1. (i) The Hilbert space $U$ is separable and satisfies $U \subset C_{b}^{1}\left(\mathbb{R}_{+}\right)$. In particular,

$$
\|\phi\|_{L^{\infty}\left(\mathbb{R}_{+}\right)}+\left\|\phi^{\prime}\right\|_{L^{\infty}\left(\mathbb{R}_{+}\right)}+\left\|\phi^{\prime}\right\|_{L^{1}\left(\mathbb{R}_{+}\right)}+\left\|\phi^{\prime \prime}\right\|_{L^{1}\left(\mathbb{R}_{+}\right)} \leqslant C\|\phi\|_{U} .
$$


(ii) The semigroup $\{S(t)\}_{t \in \mathbb{R}_{+}}$is strongly continuous on $U$, and the domain of its generator $A$ is given by $\left\{\phi \in U: \phi^{\prime} \in U\right\}$. Moreover, A satisfies $A \phi=\phi^{\prime}$.

Proof. First we will confirm that $U$ is separable. To this end, consider the Hilbert space

$$
U_{1}:=\left\{\phi \in L_{l o c}^{1}\left(\mathbb{R}_{+}\right): \text {there exists } \phi^{\prime} \in L_{l o c}^{1}\left(\mathbb{R}_{+}\right) \text {of } \phi \text { such that }\|\phi\|_{U_{1}}<\infty\right\},
$$

where

$$
\|\phi\|_{U_{1}}^{2}=|\phi(0)|^{2}+\int_{0}^{\infty}\left|\phi^{\prime}(x)\right|^{2} w(x) d x .
$$

By Theorem 5.1.1 in [8], the space $U_{1}$ is separable. Then, $U$ is isometric to a closed subspace of $U_{1} \times U_{1}$ by the mapping $\phi \mapsto\left(\phi, \phi^{\prime}\right)$. This shows that $U$ is indeed separable.

Since $\phi \in U$ has the generalized derivatives $\phi^{\prime}$ and $\phi^{\prime \prime}$, we can write

$$
\phi(x)-\phi(y)=\int_{y}^{x} \phi^{\prime}(z) d z, \quad \phi^{\prime}(x)-\phi^{\prime}(y)=\int_{y}^{x} \phi^{\prime \prime}(z) d z, \quad x, y \in \mathbb{R}_{+} .
$$

Further, as in the proof of Theorem 5.1.1 in [8], we see

$$
\left\|\phi^{\prime}\right\|_{L^{1}\left(\mathbb{R}_{+}\right)} \leqslant\left(\int_{0}^{\infty}\left|\phi^{\prime}(x)\right|^{2} w(x) d x\right)^{1 / 2}\left\|w^{-1}\right\|_{L^{1}\left(\mathbb{R}_{+}\right)}^{1 / 2} \leqslant C\|\phi\|_{U}<\infty .
$$

Combining this with (2.1), we have $\|\phi\|_{L^{\infty}\left(\mathbb{R}_{+}\right)} \leqslant C\|\phi\|_{U}$. Similarly, we see $\left\|\phi^{\prime \prime}\right\|_{L^{1}\left(\mathbb{R}_{+}\right)} \leqslant$ $C\|\phi\|_{U}$, and so $\left\|\phi^{\prime}\right\|_{L^{\infty}\left(\mathbb{R}_{+}\right)} \leqslant C\|\phi\|_{U}$. Thus the claim (i) follows.

It can be easily seen that $(S(t) \phi)^{\prime}$ and $(S(t) \phi)^{\prime \prime}$ exist and are given by $(S(t) \phi)^{\prime}(x)=$ $S(t) \phi^{\prime}(x)$ and $(S(t) \phi)^{\prime \prime}(x)=S(t) \phi^{\prime \prime}(x)$ for $\phi \in U$. Using (2.1) and the monotonicity of $w$, we find

$$
\begin{aligned}
\|S(t) \phi\|_{U}^{2} & =|\phi(t)|^{2}+\left|\phi^{\prime}(t)\right|^{2}+\int_{0}^{\infty}\left(\left|\phi^{\prime}(t+x)\right|^{2}+\left|\phi^{\prime \prime}(t+x)\right|^{2}\right) w(x) d x \\
& \leqslant C\|\phi\|_{U}^{2}+\int_{0}^{\infty}\left(\left|\phi^{\prime}(t+x)\right|^{2}+\left|\phi^{\prime \prime}(t+x)\right|^{2}\right) w(t+x) d x \leqslant C\|\phi\|_{U}^{2} .
\end{aligned}
$$

This means that $S(t)$ is bounded on $U$ for all $t \in \mathbb{R}_{+}$. To prove the strong continuity of $\{S(t)\}_{t \in \mathbb{R}_{+}}$, by the claim (i), it suffices to show that for any $t_{0} \in \mathbb{R}_{+}$and Borel measurable function $g$ on $\mathbb{R}_{+}$with $g^{2} w \in L^{1}\left(\mathbb{R}_{+}\right)$,

$$
\lim _{t \rightarrow t_{0}} \int_{0}^{\infty}\left|g(t+x)-g\left(t_{0}+x\right)\right|^{2} w(x) d x=0 .
$$

To this end, for any $\varepsilon>0$ take a bounded $E_{\varepsilon} \in \mathcal{B}\left(\mathbb{R}_{+}\right)$and a continuous function $g_{\varepsilon}$ on $\mathbb{R}_{+}$such that $g_{\varepsilon}(x)=0$ for $x \notin E_{\varepsilon}$ and that

$$
\int_{0}^{\infty}\left|g(x)-g_{\varepsilon}(x)\right|^{2} w(x) d x<\varepsilon .
$$

The existences of $E_{\varepsilon}$ and $g_{\varepsilon}$ can be proved by a routine argument in measure theory, but for completeness we give a proof later. Suppose at this moment that there exist such $E_{\varepsilon}$ 
and $g_{\varepsilon}$. Then take $\ell>0$ so that $t+E_{\varepsilon}, t_{0}+E_{\varepsilon} \subset[0, \ell]$ for $t \geqslant 0$ with $\left|t-t_{0}\right| \leqslant 1$. By the monotonicity of $w$,

$$
\begin{aligned}
& \int_{0}^{\infty}\left|g(t+x)-g\left(t_{0}+x\right)\right|^{2} w(x) d x \\
& \leqslant 3 \int_{0}^{\infty}\left|g(t+x)-g_{\varepsilon}(t+x)\right|^{2} w(x) d x+3 \int_{0}^{\infty}\left|g_{\varepsilon}(t+x)-g_{\varepsilon}\left(t_{0}+x\right)\right|^{2} w(x) d x \\
& \quad+3 \int_{0}^{\infty}\left|g_{\varepsilon}\left(t_{0}+x\right)-g\left(t_{0}+x\right)\right|^{2} w(x) d x \\
& \leqslant 3 \int_{0}^{\infty}\left|g(t+x)-g_{\varepsilon}(t+x)\right|^{2} w(t+x) d x+3 \int_{0}^{\ell}\left|g_{\varepsilon}(t+x)-g_{\varepsilon}\left(t_{0}+x\right)\right|^{2} w(x) d x \\
& \quad+3 \int_{0}^{\infty}\left|g_{\varepsilon}\left(t_{0}+x\right)-g\left(t_{0}+x\right)\right|^{2} w\left(t_{0}+x\right) d x \\
& \leqslant 6 \int_{0}^{\infty}\left|g(x)-g_{\varepsilon}(x)\right|^{2} w(x) d x+3 \sup _{x \in[0, \ell]}\left|g_{\varepsilon}(t+x)-g_{\varepsilon}\left(t_{0}+x\right)\right|^{2} \int_{0}^{\ell} w(x) d x
\end{aligned}
$$

Thus the uniform continuity of $g_{\varepsilon}$ leads to

$$
\limsup _{t \rightarrow t_{0}} \int_{0}^{\infty}\left|g(t+x)-g\left(t_{0}+x\right)\right|^{2} w(x) d x \leqslant 6 \varepsilon,
$$

whence (2.2).

To confirm the existences of $E_{\varepsilon}$ and $g_{\varepsilon}$, first notice that we can assume $g \geqslant 0$ without loss of generality. Then there exists a nondecreasing sequence of simple functions $\left\{g_{n}\right\}$ such that $g_{n}$ vanishes outside $[0, n)$ and $g_{n} \rightarrow g$ a.e. By the monotone convergence theorem, we also have

$$
\lim _{n \rightarrow \infty} \int_{0}^{\infty}\left|g(x)-g_{n}(x)\right|^{2} w(x) d x=0 .
$$

Fix $n \in \mathbb{N}$ such that

$$
\int_{0}^{\infty}\left|g(x)-g_{n}(x)\right|^{2} w(x) d x<\frac{\varepsilon}{4}
$$

Suppose that $g_{n}$ is represented as $g_{n}=\sum_{j=1}^{m} \alpha_{j} 1_{E_{j}}$. By the absolute continuity of the Lebesgue integral, for each $j=1, \ldots, m$ there exists $\delta_{j}>0$ such that for any $E^{\prime} \in \mathcal{B}([0, n))$ with $\operatorname{Leb}\left(E^{\prime}\right)<\delta_{j}$ we have

$$
\int_{E^{\prime}} w(x) d x<\frac{\varepsilon}{4 m^{2} \alpha_{j}^{2}}
$$

Now take a closed set $F_{j}$ and a open set $G_{j}$ such that $F_{j} \subset E_{j} \subset G_{j} \subset[0, n)$ with $\operatorname{Leb}\left(G_{j} \backslash F_{j}\right)<\delta_{j}$. Define the continuous function $\rho_{j}$ on $\mathbb{R}_{+}$by

$$
\rho_{j}(x)=\frac{\inf _{y \in F_{j}}|x-y|}{\inf _{y \in F_{j}}|x-y|+\inf _{y \in G_{j}^{c}}|x-y|}, \quad x \in \mathbb{R}_{+} .
$$


It is straightforward to see that

$$
\int_{0}^{\infty}\left|1_{E_{j}}(x)-\rho_{j}(x)\right|^{2} w(x) d x \leqslant \int_{G_{j} \backslash F_{j}} w(x) d x<\frac{\varepsilon}{4 m^{2} \alpha_{j}^{2}} .
$$

Thus the function $g_{\varepsilon}:=\sum_{j=1}^{m} \alpha_{j} \rho_{j}$ satisfies

$$
\int_{0}^{\infty}\left|g_{n}-g_{\varepsilon}\right|^{2} w(x) d x \leqslant m \sum_{j=1}^{m} \alpha_{j}^{2} \int_{0}^{\infty}\left|1_{E_{j}}(x)-\rho_{j}(x)\right|^{2} w(x) d x<\frac{\varepsilon}{4} .
$$

Therefore

$$
\begin{aligned}
\int_{0}^{\infty}\left|g(x)-g_{\varepsilon}(x)\right|^{2} w(x) d x & \leqslant 2 \int_{0}^{\infty}\left|g(x)-g_{n}(x)\right|^{2} w(x) d x+2 \int_{0}^{\infty}\left|g_{n}(x)-g_{\varepsilon}(x)\right|^{2} w(x) d x \\
& <\varepsilon,
\end{aligned}
$$

as claimed.

Now, as in the proof of Corollary 5.1.1 in [8], we observe, for $\phi \in U$ with $\phi^{\prime} \in U$,

$$
\begin{aligned}
& \left\|\frac{S(t) \phi-\phi}{t}-\phi^{\prime}\right\|_{U}^{2} \\
& \leqslant\left|\frac{\phi(t)-\phi(0)}{t}-\phi^{\prime}(0)\right|^{2}+\left|\frac{\phi^{\prime}(t)-\phi^{\prime}(0)}{t}-\phi^{\prime \prime}(0)\right|^{2}+2 \int_{0}^{1}\left\|S(s t) \phi^{\prime}-\phi^{\prime}\right\|_{U}^{2} d s \rightarrow 0,
\end{aligned}
$$

as $t \rightarrow 0$. Hence $A \phi=\phi^{\prime}$. Moreover, by the claim (i), the pointwise evaluation operator is continuous. This together with the strong continuity of $S$ means that the domain of $A$ is included in $\left\{\phi \in U: \phi^{\prime} \in U\right\}$ (see Lemma 4.2.2 in [8]). Thus the claim (ii) follows.

Let $\sigma_{i}, i=1, \ldots, d$, be measurable mappings from $\left(\mathbb{R}_{+} \times \Omega \times U, \mathcal{P} \otimes \mathcal{B}(U)\right)$ into $(U, \mathcal{B}(U))$, where $\mathcal{P}$ denotes the predictable $\sigma$-field and $\mathcal{B}(U)$ is the Borel $\sigma$-field of $U$, such that $\lim _{x \rightarrow \infty} \sigma_{i}(t, \omega, \phi)(x)=0$ for every $i=1, \ldots, d, t \in \mathbb{R}_{+}, \omega \in \Omega$, and $\phi \in U$. Further, we assume that the following hold:

Assumption 2.2. There exists a constant $C_{1} \in(0, \infty)$ such that for $i=1, \ldots, d$ and $(t, \omega, \phi, \psi) \in \mathbb{R}_{+} \times \Omega \times U \times U$,

$$
\begin{gathered}
\left\|\sigma_{i}(t, \omega, \phi)\right\|_{U} \leqslant C_{1} \\
\left\|\sigma_{i}(t, \omega, \phi)-\sigma_{i}(t, \omega, \psi)\right\|_{U} \leqslant C_{1}\|\phi-\psi\|_{U} .
\end{gathered}
$$

Define the mapping $\alpha$ defined on $\mathbb{R}_{+} \times \Omega \times U$ by

$$
\alpha(t, \omega, \phi)(x):=\sum_{j=1}^{d} \sigma_{j}(t, \phi)(x) \int_{0}^{x} \sigma_{j}(t, \phi)(y) d y, \quad x \in \mathbb{R}_{+} .
$$

Then we have the following: 
Lemma 2.3. Under Assumption 2.2, the mapping $\alpha$ is measurable from $\left(\mathbb{R}_{+} \times \Omega \times\right.$ $U, \mathcal{P} \otimes \mathcal{B}(U))$ into $(U, \mathcal{B}(U))$. Moreover there exists a constant $C_{2} \in(0, \infty)$ such that for $(t, \omega, \phi, \psi) \in \mathbb{R}_{+} \times \Omega \times U \times U$,

$$
\begin{gathered}
\|\alpha(t, \omega, \phi)\|_{U} \leqslant C_{2}, \\
\|\alpha(t, \omega, \phi)-\alpha(t, \omega, \psi)\|_{U} \leqslant C_{2}\|\phi-\psi\|_{U} .
\end{gathered}
$$

Proof. We consider the functional $\mathcal{S}$ on $U$ defined by

$$
\mathcal{S} \phi(x)=\phi(x) \int_{0}^{x} \phi(y) d y, \quad x \in \mathbb{R}_{+} .
$$

Then, from the proof of Theorem 5.1.1 in [8] we have

$$
\left\|(\phi-\phi(\infty))^{4} w\right\|_{L^{1}\left(\mathbb{R}_{+}\right)} \leqslant C\|\phi\|_{U}^{4}, \quad \phi \in U .
$$

Using this we obtain for $\phi \in U$ with $\phi(\infty)=0$,

$$
\begin{aligned}
& \|\mathcal{S} \phi\|_{U}^{2} \\
& =\left|\phi^{2}(0)\right|^{2}+\int_{0}^{\infty}\left(\phi^{\prime}(x) \int_{0}^{x} \phi(y) d y+\phi^{2}(x)\right)^{2} w(x) d x \\
& \quad+\int_{0}^{\infty}\left(\phi^{\prime \prime}(x) \int_{0}^{x} \phi(y) d y+\left(\phi^{\prime}(x)\right)^{2}+2 \phi(x) \phi^{\prime}(x)\right)^{2} w(x) d x \\
& \leqslant|\phi(0)|^{4}+\int_{0}^{\infty}\left(\left(2\left|\phi^{\prime}(x)\right|^{2}+3\left|\phi^{\prime \prime}(x)\right|^{2}\right)\left(\int_{0}^{x} \phi(y) d y\right)^{2}+5 \phi^{4}(x)+6\left(\phi^{\prime}(x)\right)^{4}\right) w(x) d x \\
& \leqslant\|\phi\|_{L^{\infty}\left(\mathbb{R}_{+}\right)}^{4}+5\|\phi\|_{U}^{2}\|\phi\|_{L^{1}\left(\mathbb{R}_{+}\right)}^{2}+5\|\phi\|_{U}^{4}+6\left\|\phi^{\prime}\right\|_{L^{\infty}\left(\mathbb{R}_{+}\right)}^{2}\|\phi\|_{U^{2}}^{2} .
\end{aligned}
$$

Using Proposition 2.1, we obtain $\|\mathcal{S} \phi\|_{U}^{2} \leqslant C\|\phi\|_{U}^{4}$ for $\phi \in U$ with $\phi(\infty)=0$. This and the boundedness of $\sigma_{i}$ yield, for $\phi \in U$,

$$
\|\alpha(t, \phi)\|_{U} \leqslant \sum_{i=1}^{d}\left\|\mathcal{S} \sigma_{i}(t, \phi)\right\|_{U} \leqslant C \sum_{i=1}^{d}\left\|\sigma_{i}(t, \phi)\right\|_{U}^{2} \leqslant C .
$$

In particular, $\alpha$ is measurable and $U$-valued.

Next, for $\phi, \psi \in U$, observe $\|\mathcal{S} \phi-\mathcal{S} \psi\|_{U}^{2}=I_{1}+I_{2}+I_{3}$, where

$$
\begin{aligned}
& I_{1}=\left|\phi^{2}(0)-\psi^{2}(0)\right|^{2}, \\
& I_{2}= \int_{0}^{\infty}\left\{\phi^{\prime}(x) \int_{0}^{x} \phi(y) d y+\phi^{2}(x)-\psi^{\prime}(x) \int_{0}^{x} \psi(y) d y-\psi(y)^{2}\right\}^{2} w(x) d x, \\
& I_{3}=\int_{0}^{\infty}\left\{\phi^{\prime \prime}(x) \int_{0}^{x} \phi(y) d y+\phi^{\prime}(x)^{2}+2 \phi(x) \phi^{\prime}(x)-\psi^{\prime \prime}(x) \int_{0}^{x} \psi(y) d y-\psi^{\prime}(y)^{2}\right. \\
&\left.\quad-2 \psi(x) \psi^{\prime}(x)\right\}^{2} w(x) d x .
\end{aligned}
$$


By Proposition 2.1 (i), we have

$$
I_{1}=(\phi(0)+\psi(0))^{2}(\phi(0)-\psi(0))^{2} \leqslant 2\left(\|\phi\|_{U}^{2}+\|\psi\|_{U}^{2}\right)\|\phi-\psi\|_{U}^{2} .
$$

Then, by Corollary 5.1.2 in [8],

$$
I_{2} \leqslant C\left(\|\phi\|_{U}^{2}+\|\psi\|_{U}^{2}\right)\|\phi-\psi\|_{U}^{2}
$$

Further, straightforward estimates and Proposition 2.1 (i) yield

$$
\begin{aligned}
I_{3} \leqslant & 5 \int_{0}^{\infty}\left\{\left|\phi^{\prime \prime}(x)\right|^{2}\left|\int_{0}^{x}(\phi(y)-\psi(y)) d y\right|^{2}+\left|\phi^{\prime \prime}(x)-\psi^{\prime \prime}(x)\right|^{2}\left|\int_{0}^{x} \psi(y) d y\right|^{2}\right. \\
& +\left(\phi^{\prime}(x)+\psi^{\prime}(x)\right)^{2}\left(\phi^{\prime}(x)-\psi^{\prime}(x)\right)^{2}+4|\phi(x)|^{2}\left(\phi^{\prime}(x)-\psi^{\prime}(x)\right)^{2} \\
& \left.+4\left|\psi^{\prime}(x)\right|^{2}(\phi(x)-\psi(x))^{2}\right\} w(x) d x \\
\leqslant & 5\|\phi\|_{U}^{2}\|\phi-\psi\|_{L^{1}\left(\mathbb{R}_{+}\right)}^{2}+5\|\phi-\psi\|_{U}^{2}\|\psi\|_{L^{1}\left(\mathbb{R}_{+}\right)}^{2}+5\|\phi+\psi\|_{U}^{2}\|\phi-\psi\|_{L^{\infty}\left(\mathbb{R}_{+}\right)}^{2} \\
& +20\|\phi\|_{U}^{2}\left\|\phi^{\prime}-\psi^{\prime}\right\|_{L^{\infty}\left(\mathbb{R}_{+}\right)}^{2}+20\|\psi\|_{U}^{2}\|\phi-\psi\|_{L^{\infty}\left(\mathbb{R}_{+}\right)}^{2} \\
\leqslant & C\left(\|\phi\|_{U}^{2}+\|\psi\|_{U}^{2}\right)\|\phi-\psi\|_{U}^{2} .
\end{aligned}
$$

Therefore we have

$$
\|\mathcal{S} \phi-\mathcal{S} \psi\|_{U}^{2} \leqslant C\left(\|\phi\|_{U}^{2}+\|\psi\|_{U}^{2}\right)\|\phi-\psi\|_{U}^{2} .
$$

This together with the assumptions for $\sigma_{i}$ leads to the Lipschitz continuity of $\alpha$.

Then, by Theorem 7.2 in Da Prato and Zabczyk [6], for a given $r_{0} \in U$, there exists a unique $U$-valued predictable process $r(t)=r(t, \cdot), t \in \mathbb{R}_{+}$, that is a mild solution to

$$
\left\{\begin{array}{l}
d r(t)=(A r(t)+\alpha(t, r(t))) d t+\sum_{i=1}^{d} \sigma_{i}(t, r(t)) d W_{i}(t), \\
r(0)=r_{0} .
\end{array}\right.
$$

Moreover $\{r(t)\}_{t \geqslant 0}$ has a continuous modification and satisfies

$$
\sup _{0 \leqslant t \leqslant T} \mathbb{E}\|r(t)\|_{U}^{2} \leqslant C_{T}\left(1+\left\|r_{0}\right\|_{U}^{2}\right)
$$

for some positive constant $C_{T}$ for any $T>0$. Therefore, for $t \in \mathbb{R}_{+}$,

$$
r(t)=S(t) r_{0}+\int_{0}^{t} S(t-s) \alpha(s, r(s)) d s+\sum_{i=1}^{d} \int_{0}^{t} S(t-s) \sigma_{i}(s, r(s)) d W_{i}(s), \quad \text { a.s. }
$$

Now, let $P(t, T)$ be the price at time $t$ of a discounted bond with maturity $T \geqslant t$. We assume that

$$
P(t, T)=\exp \left(-\int_{0}^{T-t} r(t, x) d x\right), \quad 0 \leqslant t \leqslant T<\infty .
$$


Then the process $f(t, T):=r(t, T-t), 0 \leqslant t \leqslant T<\infty$, satisfies

$$
f(t, T)=-\frac{\partial}{\partial T} \log P(t, T), \quad 0 \leqslant t \leqslant T<\infty,
$$

and so is interpreted as the forward rate process. If we set by abuse of notation $\sigma_{i}(t, T, \omega)=\sigma_{i}(t, \omega, r(t))(T-t)$ and $\alpha(t, T, \omega)=\alpha(t, \omega, r(t))(T-t)$, then by (2.5),

$$
\begin{aligned}
f(t, T)= & S(t) r_{0}(T-t)+\int_{0}^{t} S(t-s) \alpha(s, s+T-t) d s \\
& +\sum_{i=1}^{d} \int_{0}^{t} S(t-s) \sigma_{i}(s, s+T-t) d W_{i}(s) \\
= & r_{0}(T)+\int_{0}^{t} \alpha(s, T) d s+\sum_{i=1}^{d} \int_{0}^{t} \sigma_{i}(s, T) d W_{i}(s) .
\end{aligned}
$$

This is nothing but an HJM model for the forward rate. Further, let $\{B(t)\}_{t \in \mathbb{R}_{+}}$be the bank account process defined by

$$
B(t)=\exp \left(\int_{0}^{t} r(s, 0) d s\right), \quad t \in \mathbb{R}_{+} .
$$

Then, since the definition of $\alpha$ excludes arbitrage opportunities, $\mathbb{P}$ is an equivalent local martingale measure, i.e., the process $\{P(t, T) / B(t)\}_{0 \leqslant t \leqslant T}$ is a local martingale under $\mathbb{P}$ for any $T>0$. Consequently, the infinite dimensional SDE (2.3) leads to a risk-neutral modeling of interest rate processes.

\section{Collocation methods for HJMM equations}

In this section, we describe an approximation method for the equation (2.3) based on the kernel-based interpolation theory, and derive its error bound.

Let $\Phi: \mathbb{R} \rightarrow \mathbb{R}$ be a radial and positive definite function, i.e., $\Phi(\cdot)=\phi(|\cdot|)$ for some $\phi:[0, \infty) \rightarrow \mathbb{R}$ and for every $\ell \in \mathbb{N}$, for all pairwise distinct $y_{1}, \ldots, y_{\ell} \in \mathbb{R}$ and for all $\alpha=\left(\alpha_{i}\right) \in \mathbb{R}^{\ell} \backslash\{0\}$, we have

$$
\sum_{i, j=1}^{\ell} \alpha_{i} \alpha_{j} \Phi\left(y_{i}-y_{j}\right)>0 .
$$

Then, by Theorems 10.10 and 10.11 in Wendland [23], there exists a unique Hilbert space $\mathcal{N}_{\Phi}(\mathbb{R})$ with norm $\|\cdot\|_{\mathcal{N}_{\Phi}(\mathbb{R})}$, called the native space, of real-valued functions on $\mathbb{R}$ such that $\Phi$ is a reproducing kernel for $\mathcal{N}_{\Phi}(\mathbb{R})$.

Let $\Gamma=\left\{x_{1}, \cdots, x_{N}\right\}$ be a finite subset of $(0, \infty)$ such that $0<x_{1}<\cdots<x_{N}$ and put $K=\left\{\Phi\left(x_{i}-x_{j}\right)\right\}_{1 \leqslant i, j \leqslant N}$. Then $K$ is invertible and thus for any $g: \mathbb{R}_{+} \rightarrow \mathbb{R}$ the function

$$
I(g)(x)=\sum_{j=1}^{N}\left(\left.K^{-1} g\right|_{\Gamma}\right)_{j} \Phi\left(x-x_{j}\right), \quad x \in \mathbb{R}_{+},
$$


interpolates $g$ on $\Gamma$.

We adopt the so-called Wendland kernel for $\Phi$, which is defined as follows: for a given $\tau \in \mathbb{N} \cup\{0\}$, set the function $\Phi_{\tau}$ satisfying $\Phi_{\tau}(x)=\phi_{\tau}(|x|), x \in \mathbb{R}^{d}$, where

$$
\phi_{\tau}(r)=\int_{r}^{\infty} r_{\tau} \int_{r_{\tau}}^{\infty} r_{\tau-1} \int_{r_{\tau-1}}^{\infty} \cdots r_{2} \int_{r_{2}}^{\infty} r_{1} \max \left\{1-r_{1}, 0\right\}^{\nu} d r_{1} d r_{2} \cdots d r_{\tau}, \quad r \geqslant 0
$$

for $\tau \geqslant 1$ and $\phi_{\tau}(|x|)=\max \{1-r, 0\}^{\tau+1}$ for $\tau=0$ with $\nu=\tau+1$. Then, it follows from Theorems 9.12 and 9.13 in [23] that the function $\phi_{\tau}$ is represented as

$$
\phi_{\tau}(r)= \begin{cases}p_{\tau}(r), & 0 \leqslant r \leqslant 1 \\ 0, & r>1\end{cases}
$$

where $p_{\tau}$ is a univariate polynomial with degree $\nu+2 \tau$ having representation

$$
p_{\tau}(r)=\sum_{j=0}^{\nu+2 \tau} d_{j, \tau}^{(\nu)} r^{j}
$$

The coefficients in (3.1) are given by

$$
\begin{aligned}
d_{j, 0}^{(\nu)} & =(-1)^{j} \frac{\nu !}{j !(\nu-j) !}, \quad 0 \leqslant j \leqslant \ell, \\
d_{0, s+1}^{(\nu)} & =\sum_{j=0}^{\nu+2 s} \frac{d_{j, s}^{(\nu)}}{j+2}, \quad d_{1, s+1}^{(\nu)}=0, \quad s \geqslant 0, \\
d_{j, s+1}^{(\nu)} & =-\frac{d_{j-2, s}^{(\nu)}}{j}, \quad s \geqslant 0, \quad 2 \leqslant j \leqslant \nu+2 s+2,
\end{aligned}
$$

in a recursive way for $0 \leqslant s \leqslant \tau-1$. Further, it is known that

$$
\phi_{\tau}(r) \doteq \begin{cases}\int_{r}^{1} s(1-s)^{\tau+2}\left(s^{2}-r^{2}\right)^{\tau-1} d s, & 0 \leqslant r \leqslant 1 \\ 0, & r>1,\end{cases}
$$

where $\doteq$ denotes equality up to a positive constant factor (see Chernih et.al [4]). For example,

$$
\begin{aligned}
& \phi_{2}(r) \doteq \max \{1-r, 0\}^{5}\left(8 r^{2}+5 r+1\right) \\
& \phi_{3}(r) \doteq \max \{1-r, 0\}^{7}\left(21 r^{3}+19 r^{2}+7 r+1\right) \\
& \phi_{4}(r) \doteq \max \{1-r, 0\}^{9}\left(384 r^{4}+453 r^{3}+237 r^{2}+63 r+7\right)
\end{aligned}
$$

The function $\Phi_{\tau}$ is $C^{2 \tau}$ on $\mathbb{R}$, and the native space $\mathcal{N}_{\Phi_{\tau}}(\mathbb{R})$ coincides with $H^{\tau+1}(\mathbb{R})$ where $H^{\theta}(\mathbb{R})$ is the Sobolev space on $\mathbb{R}$ of order $\theta \geqslant 0$ based on $L^{2}$-norm. Further, the native space norm $\|\cdot\|_{\mathcal{N}_{\Phi}(\mathbb{R})}$ and the Sobolev norm $\|\cdot\|_{H^{\tau+1}(\mathbb{R})}$ are equivalent. 
In what follows, we fix $\tau \in \mathbb{N}$ and $\Phi=\Phi_{\tau}$. Further we assume that $\Gamma \subset(0, R)$ for some $R>0$. Since we can expect that

$$
\begin{gathered}
r(t, x) \approx I(r(t, \cdot))(x)=\sum_{j=1}^{N}\left(\left.K^{-1} r(t, \cdot)\right|_{\Gamma}\right)_{j} \Phi\left(x-x_{j}\right), \\
\frac{\partial}{\partial x} r(t, x) \approx \sum_{j=1}^{N}\left(K^{-1} r(t)\right)_{j} \Phi^{\prime}\left(x-x_{j}\right),
\end{gathered}
$$

possibly we have

$$
d r(t) \simeq\{A I(r(t))+\alpha(t, I(r(t)))\} d t+\sum_{i=1}^{d} \sigma_{i}(t, I(r(t))) d W_{i}(t) .
$$

Notice that the right-hand side in the equality just above allows for a finite dimensional realization. Let us now assume that $\Gamma$ and $R$ are described by a single parameter $h>0$. Let $0=t_{0}<t_{1}<\cdots<t_{n}=T$ and denote by $r^{h}\left(t_{k}, x\right), k=0, \ldots, n, x \in \mathbb{R}_{+}$, the process obtained by Euler-Maruyama approximation of the SDE above, i.e.,

$$
\begin{aligned}
r^{h}\left(t_{k+1}, x\right)= & r^{h}\left(t_{k}, x\right)+\left\{\frac{d}{d x} I\left(r^{h}\left(t_{k}\right)\right)(x)+\alpha\left(t_{k}, I\left(r^{h}\left(t_{k}\right)\right)(x)\right\} \Delta t_{k+1}\right. \\
& +\sum_{i=1}^{d} \sigma_{i}\left(t_{k}, I\left(r^{h}\left(t_{k}\right)\right)\right)(x) \Delta W_{i}\left(t_{k+1}\right), \quad k=0, \ldots, n-1, \\
r^{h}\left(t_{0}, x\right)= & r_{0}(x),
\end{aligned}
$$

where $\Delta t_{k+1}=t_{k+1}-t_{k}$ and $\Delta W_{i}\left(t_{k+1}\right)=W_{i}\left(t_{k+1}\right)-W_{i}\left(t_{k}\right)$. For $t \in\left[t_{k}, t_{k+1}\right]$ we set

$$
\begin{aligned}
r^{h}(t, x)= & r^{h}\left(t_{k}, x\right)+\left\{\frac{d}{d x} I\left(r^{h}\left(t_{k}\right)\right)(x)+\alpha\left(t_{k}, I\left(r^{h}\left(t_{k}\right)\right)(x)\right\}\left(t-t_{k}\right)\right. \\
& +\sum_{i=1}^{d} \sigma_{i}\left(t_{k}, I\left(r^{h}\left(t_{k}\right)\right)\right)(x)\left(W_{i}(t)-W_{i}\left(t_{k}\right)\right) .
\end{aligned}
$$

Now denote $I(v)(x)=\sum_{j=1}^{N}\left(K^{-1} v\right)_{j} \Phi\left(x-x_{j}\right)$ for $v \in \mathbb{R}^{N}$ by an abuse of notation, and set

$$
\begin{aligned}
& \alpha(t, v)=\left(\alpha(t, I(v))\left(x_{1}\right), \ldots, \alpha(t, I(v))\left(x_{N}\right)\right)^{\mathrm{T}}, \\
& \sigma(t, v)=\left\{\sigma_{i}(t, I(v))\left(x_{j}\right)\right\}_{\substack{1 \leqslant j \leqslant N \\
1 \leqslant i \leqslant d}} \in \mathbb{R}^{N \times d}, \quad(t, v) \in[0, T] \times \mathbb{R}^{N} .
\end{aligned}
$$

Also, notice that by setting $K_{1}=\left\{\Phi^{\prime}\left(x_{j}-x_{\ell}\right)\right\}_{1 \leqslant j, \ell \leqslant N}$, we obtain $I^{\prime}(\phi)\left(x_{j}\right)=\left(\left.K_{1} K^{-1} \phi\right|_{\Gamma}\right)_{j}$. Then, $r_{k}^{h}:=\left(r^{h}\left(t_{k}, x_{1}\right), \ldots, r^{h}\left(t_{k}, x_{N}\right)\right)^{\top} \in \mathbb{R}^{N}, k=0, \ldots, n$, is given by

$$
r_{k+1}^{h}=r_{k}^{h}+\left(K_{1} K^{-1} r_{k}^{h}+\alpha\left(t_{k}, r_{k}^{h}\right)\right) \Delta t_{k+1}+\sigma\left(t_{k}, r_{k}^{h}\right) \Delta W\left(t_{k+1}\right)
$$


with $r_{0}^{h}=\left(r_{0}\left(x_{1}\right), \ldots, r_{0}\left(x_{N}\right)\right)^{\top}$, which is the Euler-Maruyama approximation of the $N$-dimensional stochastic differential equation

$$
\left\{\begin{aligned}
d \tilde{r}(t) & =\left[K_{1} K^{-1} \tilde{r}(t)+\alpha(t, \tilde{r}(t))\right] d t+\sigma(t, \tilde{r}(t)) d W(t), \\
\tilde{r}(0) & =\left(r_{0}\left(x_{1}\right), \ldots, r_{0}\left(x_{N}\right)\right)^{\top}
\end{aligned}\right.
$$

Furthermore, suppose that we compute $r^{h}\left(t_{k}, \cdot\right)$ at points in $\Gamma_{e}=\left\{\xi_{1}, \ldots, \xi_{M}\right\} \subset[0, \infty)$. Then, $\tilde{r}_{k}^{h}:=\left(r^{h}\left(t_{k}, \xi_{1}\right), \ldots, r^{h}\left(t_{k}, \xi_{M}\right)\right)^{\top}, k=0, \ldots, n$, is given by

$$
\tilde{r}_{k+1}^{h}=\tilde{r}_{k}^{h}+\left(K_{1 e} K^{-1} r_{k}^{h}+\tilde{\alpha}\left(t_{k}, r_{k}^{h}\right)\right) \Delta t_{k+1}+\tilde{\sigma}\left(t_{k}, r_{k}^{h}\right) \Delta W\left(t_{k+1}\right)
$$

with $\tilde{r}_{0}^{h}=\left(r_{0}\left(\xi_{1}\right), \ldots, r_{0}\left(\xi_{M}\right)\right)^{\top}$, where $K_{1 e}=\left\{\Phi^{\prime}\left(\xi_{j}-x_{\ell}\right)\right\}_{1 \leqslant j \leqslant M, 1 \leqslant \ell \leqslant N}$,

$$
\begin{aligned}
& \tilde{\alpha}(t, v)=\left(\alpha(t, I(v))\left(\xi_{1}\right), \ldots, \alpha(t, I(v))\left(\xi_{M}\right)\right)^{\mathrm{T}}, \\
& \tilde{\sigma}(t, v)=\left\{\sigma_{i}(t, I(v))\left(\xi_{j}\right)\right\}_{\substack{1 \leqslant j \leqslant M \\
1 \leqslant i \leqslant d}} \in \mathbb{R}^{M \times d}, \quad(t, v) \in[0, T] \times \mathbb{R}^{N} .
\end{aligned}
$$

The rest of this section is devoted to the proof of a convergence of the approximation above. To this end, we impose the following conditions on $r_{0}$ and $\sigma_{i}$ 's:

Assumption 3.1. (i) The function $r_{0}$ belongs to $U \cap C_{b}^{2}\left(\mathbb{R}_{+}\right)$.

(ii) The function $\sigma(t, \omega, \phi)$ is $C^{2}$ on $\mathbb{R}_{+}$for any $t \in \mathbb{R}_{+}, \omega \in \Omega$, and $\phi \in U$.

(iii) There exist a nonnegative and Borel measurable function $\Psi$ on $\mathbb{R}_{+}$with $\Psi^{2} w \in$ $L^{1}\left(\mathbb{R}_{+}\right), \lim _{x \rightarrow \infty} \Psi(x)=0$ and a positive constant $T_{0}$ such that for $i=1, \ldots, d$, $t, x \in \mathbb{R}_{+}, \omega \in \Omega$, and $m=0,1,2$,

$$
\left|\frac{d^{m}}{d x^{m}} \sigma_{i}(t, \omega, \phi)(x)\right| \leqslant \Psi(x),
$$

and that for $i=1, \ldots, d, t, s, x \in \mathbb{R}_{+}, \omega \in \Omega, m=0,1,2$, and $\phi, \psi \in U$,

$$
\left|\frac{d^{m}}{d x^{m}} \sigma_{i}(t, \omega, \phi)(x)-\frac{d^{m}}{d x^{m}} \sigma_{i}(s, \omega, \psi)(x)\right| \leqslant \Psi(x) \sqrt{|t-s|}+\Psi(x) \int_{0}^{T_{0}}|\phi(y)-\psi(y)| d y .
$$

Notice that Assumption 2.2 holds under Assumption 3.1 since $|\phi(y)-\psi(y)| \leqslant C \| \phi-$ $\psi \|_{U}$ by Proposition 2.1 (i). Thus there exists a unique $U$-valued predictable process $\{r(t)\}_{t \geqslant 0}$ satisfying 2.4 and $(2.5)$. Then, set

$$
\Delta t=\max _{1 \leqslant i \leqslant n}\left(t_{i}-t_{i-1}\right), \quad \Delta x=\sup _{x \in(0, R)} \min _{j=1, \ldots, N}\left|x-x_{j}\right| .
$$

Since we have assumed that $\Gamma$, and $R$ are functions of $h$, so is $\Delta x$. Moreover we assume that $\left\{t_{k}\right\}_{k=0}^{n}$ is also a function of $h$. Then so is $\Delta t$.

For $j=1, \ldots, N$, we write $Q_{j}$ for the cardinal function defined by

$$
Q_{j}(x)=\sum_{i=1}^{N}\left(K^{-1}\right)_{i j} \Phi\left(x-x_{i}\right), \quad x \in \mathbb{R}, \quad j=1, \ldots, N .
$$

In what follows, $\# \mathcal{K}$ denotes the cardinality of a finite set $\mathcal{K}$. 
Assumption 3.2. (i) The parameters $\Delta t, R, N$, and $\Delta x$ satisfy $\Delta t \rightarrow 0, R \rightarrow \infty$, $N \rightarrow \infty$, and $\Delta x \rightarrow 0$ as $h \searrow 0$.

(ii) There exist $c_{1}, c_{2}, c_{3}$, positive constants independent of $h$, such that for $m=0,1,2$,

$$
\max _{x \in \Gamma \cup \Gamma_{e}} \#\left\{j \in\{1, \ldots, N\}:\left|\frac{d^{m} Q_{j}}{d x^{m}}(x)\right|>\frac{c_{1}}{N}\right\} \leqslant c_{2} R^{1 / 2} \leqslant c_{3}(\Delta x)^{-(\tau-3 / 2)} .
$$

Remark 3.3. Suppose that $\Gamma$ is quasi-uniform in the sense that

$$
c_{4} R N^{-1} \leqslant \Delta x \leqslant c_{5} R N^{-1}
$$

hold for some positive constants $c_{4}, c_{5}$. In this case, a sufficient condition for which the latter inequality in Assumption 3.1 (ii) holds is

$$
R \leqslant c_{6} N^{(2 \tau-3) /(2 \tau-2)}
$$

for some positive constant $c_{6}$.

Theorem 3.4. Suppose that Assumptions 3.1 and 3.2 hold. Moreover assume that $\tau \geqslant 3$. Then there exists $h_{0} \in(0,1]$ such that for any $T \in(0, \infty)$ we have

$$
\mathbb{E}\left|r(t, x)-r^{h}(t, x)\right|^{2} \leqslant C \Delta t+C(\Delta x)^{(2 \tau-1) / \tau} R^{1 /(2 \tau)}, \quad x \in \Gamma \cup \Gamma_{e}, \quad 0 \leqslant t \leqslant T, \quad h \leqslant h_{0} .
$$

To prove Theorem 3.4 we need several preliminary lemmas. First, recall from [20] that for any $f: \mathbb{R} \rightarrow \mathbb{R}$,

$$
I(f)(x)=\sum_{j=1}^{N}\left(\left.K^{-1} f\right|_{\Gamma}\right)_{j} \Phi\left(x-x_{j}\right)=\sum_{j=1}^{N} f\left(x_{j}\right) Q_{j}(x), \quad x \in \mathbb{R} .
$$

We use the stability results for kernel-based interpolations as in [20].

Lemma 3.5. Suppose that Assumption 3.2 and $\tau \geqslant 3$ hold. Then, there exists $h_{0} \in(0,1]$ such that

$$
\sup _{0<h \leqslant h_{0}} \max _{x \in \Gamma \cup \Gamma_{e}} \sum_{j=1}^{N}\left|\frac{d^{m} Q_{j}}{d x^{m}}(x)\right|<\infty, \quad m=0,1,2 .
$$

Proof. We write $\tilde{x}_{j}=x_{j}-R / 2$ for $j=1, \ldots, N$ and consider $\tilde{\Gamma}=\left\{\tilde{x}_{1}, \ldots, \tilde{x}_{N}\right\} \subset$ $(-R / 2, R / 2)$. With this collocation points we have $K=\left\{\Phi\left(\tilde{x}_{i}-\tilde{x}_{j}\right)\right\}_{i, j=1, \ldots, N}$ and $I(g)(x+$ $R / 2)=\sum_{j=1}^{N}\left(\left.K^{-1} \tilde{g}\right|_{\Gamma}\right)_{j} \Phi\left(x-\tilde{x}_{j}\right)$, where $\tilde{g}(x)=g(x+R / 2)$ for $x \in \mathbb{R}$. Then we can apply Lemma 3.5 in [20] to obtain the required result.

Lemma 3.6. Suppose that Assumption 3.2 and $\tau \geqslant 3$ hold. Let $h_{0}$ as in Lemma 3.5 . Then for $h \in\left(0, h_{0}\right]$ we have

(i) for $u \in H^{\tau+1}(\mathbb{R})$

$$
\left\|\frac{d}{d x}(u-I(u))\right\|_{L^{\infty}([0, R])} \leqslant C(\Delta x)^{\tau-1}\|u\|_{H^{\tau+1}(\mathbb{R})} ;
$$


(ii) for $m=0,1$ and $u \in C_{b}^{1+m}\left(\mathbb{R}_{+}\right)$

$$
\max _{x \in \Gamma \cup \Gamma_{e}}\left|\frac{d^{m}}{d x^{m}}(u-I(u))(x)\right| \leqslant C\|u\|_{C^{1+m}\left(\mathbb{R}_{+}\right)}(\Delta x)^{(\tau+1 / 2-m) /(\tau+1-m)} R^{1 /(2(\tau+1-m)} .
$$

Proof. As in the proof of the previous lemma, we translate the approximation region and the set of collocation points to $(-R / 2, R / 2)$ and $\tilde{\Gamma}$, respectively. Then applying Lemma 3.4 in [20] to $\tilde{u}(x):=u(x+R / 2), x \in \mathbb{R}$, we obtain the claim (i).

To show the claim (ii), let $u \in C_{b}^{1+m}\left(\mathbb{R}_{+}\right)$. We define an extension $\tilde{u}$ on $\mathbb{R}$ of $u$ by

$$
\tilde{u}(x)= \begin{cases}u(x) \zeta(x), & x \geqslant 0, \\ \left(u(0)+u^{\prime}(0) x+(m / 2)\left(d^{m} u^{\prime} / d x^{m}\right)(0) x^{2}\right) \zeta(x), & x<0,\end{cases}
$$

where $\zeta$ is $C^{\infty}$-function on $\mathbb{R}$ such that $0 \leqslant \zeta \leqslant 1, \zeta=1$ on $(-\delta, \infty)$, and $\zeta=0$ on $(-\infty,-2 \delta)$, for some fixed $\delta>0$. Then it is straightforward to see that $\tilde{u}$ is $C_{b}^{1+m}(\mathbb{R})$ such that $d^{\kappa} \tilde{u} / d x^{\kappa}=d^{\kappa} u / d x^{\kappa}$ on $\mathbb{R}_{+}$for $0 \leqslant \kappa \leqslant 1+m$ and $\|\tilde{u}\|_{C^{1+m}(\mathbb{R})} \leqslant C\|u\|_{C^{1+m}\left(\mathbb{R}_{+}\right)}$. Further, take a $C^{\infty}$-function $\rho$ with a compact support and unit integral, and set $\rho_{\varepsilon}(x)=$ $(1 / \varepsilon) \rho(x / \varepsilon)$ for $x \in \mathbb{R}$ and $\varepsilon>0$. With this mollifier and the function $\tilde{u}$, we define $u_{\varepsilon}$ by

$$
u_{\varepsilon}(x)=\int_{-\infty}^{\infty} \tilde{u}(y) \rho_{\varepsilon}(x-y) d y, \quad x \in \mathbb{R} .
$$

This function satisfies

$$
\begin{aligned}
& \sup _{x \in \mathbb{R}}\left|\frac{d^{m}}{d x^{m}}\left(\tilde{u}(x)-u_{\varepsilon}\right)(x)\right| \leqslant C\|u\|_{C^{1+m}\left(\mathbb{R}_{+}\right)} \varepsilon, \\
& \sup _{x \in \mathbb{R}}\left|\frac{d^{\kappa}}{d x^{\kappa}} u_{\varepsilon}(x)\right| \leqslant C \varepsilon^{-\max \{\kappa-1-m, 0\}}\|u\|_{C^{1+m}\left(\mathbb{R}_{+}\right)}, \quad \kappa \in \mathbb{N} \cup\{0\} .
\end{aligned}
$$

We take another $C^{\infty}$-function $\zeta_{1}$ on $\mathbb{R}$ such that $0 \leqslant \zeta_{1} \leqslant 1$ on $\mathbb{R}, \zeta_{1}(x)=1$ for $|x| \leqslant 1$, and $\zeta_{1}(x)=0$ for $|x|>1+c$ for some $c>0$. Then consider the function $\hat{u}_{\varepsilon}(x):=u_{\varepsilon}(x) \zeta_{1}(x / R), x \in \mathbb{R}$. Trivially, $\hat{u}_{\varepsilon} \in H^{\tau+1}(\mathbb{R})$ and by $(3.2)$,

$$
\left\|\hat{u}_{\varepsilon}\right\|_{H^{\tau+1}(\mathbb{R})}^{2} \leqslant C \sum_{\kappa=0}^{\tau+1} \int_{-(1+c) R}^{(1+c) R}\left|\frac{d^{\kappa}}{d x^{\kappa}} u_{\varepsilon}(x)\right|^{2} d x \leqslant C R \varepsilon^{-2(\tau-m)}\|u\|_{C^{1+m}\left(\mathbb{R}_{+}\right)}^{2} .
$$

From this estimates and applying Lemma 3.6 in [20] to $\hat{u}_{\varepsilon}$, we have

$$
\begin{aligned}
\sup _{0 \leqslant x \leqslant R}\left|\frac{d^{m}}{d x^{m}}\left(\hat{u}_{\varepsilon}-I\left(\hat{u}_{\varepsilon}\right)\right)(x)\right| & \leqslant C(\Delta x)^{\tau+1 / 2-m}\left\|\hat{u}_{\varepsilon}\right\|_{H^{\tau+1}(\mathbb{R})} \\
& \leqslant C(\Delta x)^{\tau+1 / 2-m} R^{1 / 2} \varepsilon^{-(\tau-m)}\|u\|_{C^{2}\left(\mathbb{R}_{+}\right)} .
\end{aligned}
$$

This together with Lemma 3.5 leads to

$$
\begin{aligned}
& \left|\frac{d^{m}}{d x^{m}}(u-I(u))(x)\right| \\
& \leqslant\left|\frac{d^{m}}{d x^{m}}\left(\tilde{u}-u_{\varepsilon}\right)(x)\right|+\left|\frac{d^{m}}{d x^{m}}\left(\hat{u}_{\varepsilon}-I\left(\hat{u}_{\varepsilon}\right)\right)(x)\right|+\left|\frac{d^{m}}{d x^{m}}\left(I\left(u_{\varepsilon}\right)-I(\tilde{u})\right)(x)\right| \\
& \leqslant C\|u\|_{C^{2}\left(\mathbb{R}_{+}\right)} \varepsilon+C(\Delta x)^{\tau+1 / 2-m} R^{1 / 2} \varepsilon^{-(\tau-m)}\|u\|_{C^{2}\left(\mathbb{R}_{+}\right)}
\end{aligned}
$$


for all $x \in \Gamma \cup \Gamma_{e}$. Minimizing the right-hand side in the last inequality just above over $\varepsilon>0$, we obtain the claim (ii).

Lemma 3.7. Suppose that the assumptions imposed in Theorem 3.4 hold. Let $h_{0}$ as in Lemma 3.5. Then,

$$
\sup _{0<h \leqslant h_{0}} \max _{x \in \Gamma \cup \Gamma_{e}} \max _{k=0, \ldots, n} \mathbb{E}\left|r^{h}\left(t_{k}, x\right)\right|^{2}<\infty
$$

Proof. Fix $k=0,1, \ldots, n-1$ and $x \in \Gamma \cup \Gamma_{e}$. We use the representation

$$
\begin{aligned}
\left|r^{h}\left(t_{k+1}, x\right)\right|^{2}= & \left|r^{h}\left(t_{k}, x\right)\right|^{2}+\Lambda\left(t_{k}, x\right)^{2}\left(\Delta t_{k+1}\right)^{2}+\left(\sum_{i=1}^{d} \Theta_{i}\left(t_{k}, x\right) \Delta W_{i}\left(t_{k+1}\right)\right)^{2} \\
& +2 r^{h}\left(t_{k}, x\right) \Lambda\left(t_{k}, x\right) \Delta t_{k+1}+2 \Lambda\left(t_{k}, x\right) \Delta t_{k+1} \sum_{i=1}^{d} \Theta_{i}\left(t_{k}, x\right) \Delta W_{i}\left(t_{k+1}\right) \\
& +2 r^{h}\left(t_{k}, x\right) \sum_{i=1}^{d} \Theta_{i}\left(t_{k}, x\right) \Delta W_{i}\left(t_{k+1}\right)
\end{aligned}
$$

where for $i=1, \ldots, d$ and $x \in \mathbb{R}_{+}$

$$
\begin{aligned}
\Lambda\left(t_{k}, x\right) & =\frac{d}{d x} I\left(r^{h}\left(t_{k}\right)\right)(x)+\alpha\left(t_{k}, I\left(r^{h}\left(t_{k}\right)\right)(x),\right. \\
\Theta_{i}\left(t_{k}, x\right) & =\sigma_{i}\left(t_{k}, I\left(r^{h}\left(t_{k}\right)\right)\right)(x) .
\end{aligned}
$$

Using Lemma 3.5 and Assumption 3.1, we see

$$
\mathbb{E}\left[\Lambda\left(t_{k}, x\right)^{2}\left(\Delta t_{k+1}\right)^{2}\right]+\left|\mathbb{E} r^{h}\left(t_{k}, x\right) \Lambda\left(t_{k}, x\right)\right| \Delta t_{k+1} \leqslant C\left(1+\max _{y \in \Gamma \cup \Gamma_{e}} \mathbb{E}\left|r^{h}\left(t_{k}, y\right)\right|^{2}\right) \Delta t .
$$

Since $\Lambda\left(t_{k}, x\right)$ and $\Theta_{i}\left(t_{k}, x\right)$ are $\mathcal{F}_{t_{k}}$-measurable, for $i=1, \ldots, d$ and $j \neq i$,

$$
\begin{aligned}
\mathbb{E}\left[\Lambda\left(t_{k}, x\right) \Theta_{i}\left(t_{k}, x\right) \Delta W_{i}\left(t_{k+1}\right)\right] & =\mathbb{E}\left[r^{h}\left(t_{k}, x\right) \Theta_{i}\left(t_{k}, x\right) \Delta W_{i}\left(t_{k+1}\right)\right] \\
& =\mathbb{E}\left[\Theta_{i}\left(t_{k}, x\right) \Theta_{j}\left(t_{k}, x\right) \Delta W_{i}\left(t_{k+1}\right) \Delta W_{j}\left(t_{k+1}\right)\right] \\
& =0 .
\end{aligned}
$$

Moreover we obtain

$$
\mathbb{E}\left[\Theta_{i}\left(t_{k}, x\right)^{2}\left(\Delta W_{i}\left(t_{k+1}\right)\right)^{2}\right]=\mathbb{E}\left[\Theta_{i}\left(t_{k}, x\right)^{2}\right] \Delta t_{k+1} \leqslant C \Delta t, \quad i=1, \ldots, d .
$$

From this, (3.3) and (3.4) we deduce that

$$
\mathbb{E}\left|r^{h}\left(t_{k+1}, x\right)\right|^{2} \leqslant(1+C \Delta t) \max _{y \in \Gamma \cup \Gamma_{e}} \mathbb{E}\left|r^{h}\left(t_{k}, y\right)\right|^{2}+C \Delta t
$$

which leads to the required result. 
We denote $\tilde{A}=A I$, i.e., $\tilde{A} \phi(x)=I^{\prime}(\phi)(x)$ for $\phi \in U$ and $x \in \mathbb{R}_{+}$. Then, since $\Phi$ is supported in the unit ball,

$$
\begin{aligned}
\|\tilde{A} \phi\|_{U}^{2} & =\left|I^{\prime}(\phi)(0)\right|^{2}+\left|I^{\prime \prime}(\phi)(0)\right|^{2}+\int_{0}^{R+1}\left\{I^{\prime \prime}(\phi)(x)^{2}+I^{\prime \prime \prime}(\phi)(x)\right\}^{2} w(x) d x \\
& \leqslant C_{h} \max _{j=1, \ldots, N}\left|\phi\left(x_{j}\right)\right|^{2} \leqslant C_{h}\|\phi\|_{U}^{2}
\end{aligned}
$$

for some positive constant $C_{h}$ depending on $h$. Thus $\tilde{A}: U \rightarrow U$ is bounded, whence there exists a uniformly continuous semigroup $\tilde{S}$ on $U$ such that its generator is given by $\tilde{A}$.

Lemma 3.8. Suppose that Assumption 3.2 and $\tau \geqslant 3$ hold. Let $h_{0}$ as in Lemma 3.5 . Then for $h \in\left(0, h_{0}\right], T \in(0, \infty)$ and $\phi \in U \cap C_{b}^{2}\left(\mathbb{R}_{+}\right)$we have

$$
\max _{x \in \Gamma \cup \Gamma_{e}}|S(t) \phi(x)-\tilde{S}(t) \phi(x)| \leqslant C(\Delta x)^{(\tau-1 / 2) / \tau} R^{1 /(2 \tau)}\|\phi\|_{C^{2}\left(\mathbb{R}_{+}\right)}, \quad 0 \leqslant t \leqslant T .
$$

Proof. Let $\phi \in U \cap C_{b}^{2}\left(\mathbb{R}_{+}\right)$be fixed. Since $\{S(t)\}_{0 \leqslant t \leqslant T}$ and $\{\tilde{S}(t)\}_{0 \leqslant t \leqslant T}$ are both $C_{0^{-}}$ semigroups and the pointwise evaluation operator is bounded on $U$, we have

$$
S(t) \phi(x)-\tilde{S}(t) \phi(x)=\int_{0}^{t}\{A S(\tau) \phi(x)-\tilde{A} \tilde{S}(\tau) \phi(x)\} d \tau, \quad 0 \leqslant t \leqslant T .
$$

Thus,

$$
\begin{aligned}
\max _{x \in \Gamma \cup \Gamma_{e}}|S(t) \phi(x)-\tilde{S}(t) \phi(x)| \leqslant & \|\tilde{A}\|_{\Gamma \cup \Gamma_{e}} \int_{0}^{t} \max _{x \in \Gamma \cup \Gamma_{e}}|S(\tau) \phi(x)-\tilde{S}(\tau) \phi(x)| d \tau \\
& +\int_{0}^{t} \max _{x \in \Gamma \cup \Gamma_{e}}|A S(\tau) \phi(x)-\tilde{A} S(\tau) \phi(x)| d \tau
\end{aligned}
$$

where for $\mathcal{S}: U \rightarrow U$,

$$
\|\mathcal{S}\|_{\Gamma \cup \Gamma_{e}}=\sup \left\{\max _{x \in \Gamma \cup \Gamma_{e}}|\mathcal{S} \psi(x)| / \max _{x \in \Gamma \cup \Gamma_{e}}|\psi(x)|: \psi \in U, \max _{x \in \Gamma \cup \Gamma_{e}}|\psi(x)|>0\right\} .
$$

Lemmas 3.5 and 3.6 now imply that $\sup _{h}\|\tilde{A}\|_{\Gamma \cup \Gamma_{e}}$ is finite, and

$$
\begin{aligned}
\max _{x \in \Gamma \cup \Gamma_{e}}|A S(\tau) \phi(x)-\tilde{A} S(\tau) \phi(x)| & \leqslant C(\Delta x)^{(\tau-1 / 2) / \tau} R^{1 /(2 \tau)}\|S(\tau) \phi\|_{C^{2}\left(\mathbb{R}_{+}\right)} \\
& \leqslant C(\Delta x)^{(\tau-1 / 2) / \tau} R^{1 /(2 \tau)}\|\phi\|_{C^{2}\left(\mathbb{R}_{+}\right)}, \quad 0 \leqslant \tau \leqslant T .
\end{aligned}
$$

Thus by Gronwall's lemma, the lemma follows.

Proof of Theorem 3.4. First notice that $r^{h}(t, x)$ can be written as

$$
\begin{aligned}
r^{h}(t, x)= & r_{0}(x)+\int_{0}^{t}\left\{\tilde{A} r^{h}(s, x)+\alpha\left(s, I\left(r^{h}(s)\right)\right)(x)\right\} d s+\sum_{i=1}^{d} \int_{0}^{t} \sigma_{i}\left(s, I\left(r^{h}(s)\right)\right)(x) d W_{i}(s) \\
& -\sum_{i=0}^{d} \int_{0}^{t} \Theta_{i}(s, x) d W_{i}(s),
\end{aligned}
$$


where $W_{0}(t)=t$ and

$$
\begin{aligned}
& \Theta_{0}(s, x)=\sum_{k=0}^{n-1}\left\{\frac{d}{d x} I\left(r^{h}(s)-r^{h}\left(t_{k}\right)\right)(x)+\alpha\left(s, I\left(r^{h}(s)\right)\right)(x)-\alpha\left(t_{k}, I\left(r^{h}\left(t_{k}\right)\right)\right)(x)\right\} 1_{\left(t_{k}, t_{k+1}\right]}(s), \\
& \Theta_{i}(s, x)=\sum_{k=0}^{n-1}\left(\sigma_{i}\left(s, I\left(r^{h}(s)\right)\right)-\sigma_{i}\left(t_{k}, I\left(r^{h}\left(t_{k}\right)\right)\right)(x)\right) 1_{\left(t_{k}, t_{k+1}\right]}(s), \quad i=1, \ldots, d .
\end{aligned}
$$

This means that $r^{h}$ is a mild solution to the corresponding equation, whence

$$
\begin{aligned}
r^{h}(t)= & \tilde{S}(t) r_{0}+\int_{0}^{t} \tilde{S}(t-s)\left(\alpha\left(s, I\left(r^{h}(s)\right)\right)-\Theta_{0}(s)\right) d s \\
& +\sum_{i=1}^{d} \int_{0}^{t} \tilde{S}(t-s)\left(\sigma_{i}\left(s, I\left(r^{h}(s)\right)\right)-\Theta_{i}(s)\right) d W_{i}(s) .
\end{aligned}
$$

Thus

$$
\begin{aligned}
& r(t)-r^{h}(t) \\
& =(S(t)-\tilde{S}(t)) r_{0}+\int_{0}^{t}\left\{S(t-s) \alpha(s, r(s))-\tilde{S}(t-s) \alpha\left(s, I\left(r^{h}(s)\right)\right)+\tilde{S}(t-s) \Theta_{0}(s)\right\} d s \\
& \quad+\sum_{i=1}^{d} \int_{0}^{t}\left\{S(t-s) \sigma_{i}(s, r(s))-\tilde{S}(t-s) \sigma_{i}\left(s, I\left(r^{h}(s)\right)\right)+\tilde{S}(t-s) \Theta_{i}(s)\right\} d W_{i}(s),
\end{aligned}
$$

and so, for a fixed $x \in \Gamma \cup \Gamma_{e}$,

$$
\mathbb{E}\left|r(t, x)-r^{h}(t, x)\right|^{2} \leqslant C\left(\mathcal{I}_{1}+\mathcal{I}_{2}+\mathcal{I}_{3}+\mathcal{I}_{4}+\mathcal{I}_{5}+\mathcal{I}_{6}\right),
$$

where

$$
\begin{aligned}
& \mathcal{I}_{1}=\left|S(t) r_{0}(x)-\tilde{S}(t) r_{0}(x)\right|^{2}, \\
& \mathcal{I}_{2}=\mathbb{E} \int_{0}^{t}|S(t-s) \alpha(s, r(s))(x)-\tilde{S}(t-s) \alpha(s, r(s))(x)|^{2} d s \\
& \mathcal{I}_{3}=\mathbb{E} \int_{0}^{t}|\tilde{S}(t-s) \alpha(s, r(s))(x)-\tilde{S}(t-s) \alpha(s, I(r(s)))(x)|^{2} d s, \\
& \mathcal{I}_{4}=\mathbb{E} \int_{0}^{t}\left|\tilde{S}(t-s) \alpha(s, I(r(s)))(x)-\tilde{S}(t-s) \alpha\left(s, I\left(r^{h}(s)\right)\right)(x)\right|^{2} d s, \\
& \mathcal{I}_{5}=\mathbb{E} \int_{0}^{t}\left|\tilde{S}(t-s) \Theta_{0}(s)\right|^{2} d s, \\
& \mathcal{I}_{6}=\sum_{i=1}^{d} \mathbb{E} \int_{0}^{t}\left|S(t-s) \sigma_{i}(s, r(s))(x)-\tilde{S}(t-s) \sigma_{i}\left(s, I\left(r^{h}(s)\right)\right)(x)-\tilde{S}(t-s) \Theta_{i}(s)\right|^{2} d s .
\end{aligned}
$$

By Lemma 3.8,

$$
\max _{y \in \Gamma \cup \Gamma_{e}}\left|S(t) r_{0}(y)-\tilde{S}(t) r_{0}(y)\right|^{2} \leqslant C(\Delta x)^{(2 \tau-1) / \tau} R^{1 / \tau}
$$


Using Assumption 3.1, we observe, for $t, s \in[0, T]$ and $\phi, \psi \in U$,

$$
\begin{gathered}
\|\alpha(t, \phi)\|_{C^{2}\left(\mathbb{R}_{+}\right)} \leqslant C, \\
\sup _{y \in \mathbb{R}_{+}} \| \alpha(t, \phi)(y)-\alpha(s, \psi)(y)\left|\leqslant C \sqrt{|t-s|}+C \int_{0}^{T_{0}}\right| \phi(y)-\psi(y) \mid d y .
\end{gathered}
$$

From $\sup _{h}\|\tilde{A}\|_{\Gamma \cup \Gamma_{e}}<\infty$, we find

$$
\sup _{0 \leqslant \tau \leqslant T} \sup _{0<h \leqslant h_{0}}\|\tilde{S}(\tau)\|_{\Gamma \cup \Gamma_{e}}<\infty .
$$

Also, since $r(s) \in C_{b}^{1}\left(\mathbb{R}_{+}\right)$, we can apply Lemma 3.6 to obtain

$$
|r(s)(x)-I(r(s))(x)| \leqslant C\|r(s)\|_{U}(\Delta x)^{(\tau-1 / 2) / \tau} R^{1 /(2 \tau)}, \quad x \in \Gamma \cup \Gamma_{e}, \quad 0 \leqslant s \leqslant T .
$$

By Lemmas 3.5 and 3.8, (3.8)-(3.10) and (2.4), we see

$$
\begin{gathered}
\mathcal{I}_{2} \leqslant C(\Delta x)^{(2 \tau-1) / \tau} R^{1 / \tau} \\
\mathcal{I}_{3} \leqslant C \int_{0}^{t}\|\tilde{S}(t-s)\|_{\Gamma \cup \Gamma_{e}} \int_{0}^{T_{0}} \mathbb{E}|r(s, y)-I(r(s))(y)|^{2} d y d s \leqslant C(\Delta x)^{(2 \tau-1) / \tau} R^{1 / \tau},
\end{gathered}
$$

and

$$
\begin{aligned}
\mathcal{I}_{4} & \leqslant C \int_{0}^{t}\|\tilde{S}(t-s)\|_{\Gamma \cup \Gamma_{e}}^{2} \int_{0}^{T_{0}} \mathbb{E}\left|I\left(r(s)-r^{h}(s)\right)(y)\right|^{2} d y d s \\
& \leqslant C \int_{0}^{t} \max _{y \in \Gamma \cup \Gamma_{e}} \mathbb{E}\left|r(s, y)-r^{h}(s, y)\right|^{2} d s .
\end{aligned}
$$

Further, by (3.9),

$$
\begin{aligned}
& \int_{0}^{t}\left|\tilde{S}(t-s) \Theta_{0}(s)\right|^{2} d s \\
& =\sum_{k=0}^{n-1} \int_{t_{k} \wedge t}^{t_{k+1} \wedge t}\left|\tilde{S}(t-s) \Theta_{0}(s)\right|^{2} d s \\
& =\sum_{k=0}^{n-1} \int_{t_{k} \wedge t}^{t_{k+1} \wedge t} \mid \tilde{S}(t-s)\left(\alpha\left(s, I\left(r^{h}(s)\right)\right)(x)-\left.\alpha\left(t_{k}, I\left(r^{h}\left(t_{k}\right)\right)\right)(x)\right|^{2} d s\right. \\
& \leqslant C \sup _{0 \leqslant \tau \leqslant T}\|\tilde{S}(\tau)\|_{\Gamma \cup \Gamma_{e}} \sum_{k=0}^{n-1} \int_{t_{k} \wedge t}^{t_{k+1} \wedge t}\left\{s-t_{k}+\int_{0}^{T_{0}}\left|I\left(r^{h}(s)\right)(y)-I\left(r^{h}\left(t_{k}\right)\right)(y)\right|^{2} d y\right\} d s .
\end{aligned}
$$

Taking the expectation, we obtain

$$
\begin{aligned}
\mathbb{E} \int_{0}^{t}\left|\tilde{S}(t-s) \Theta_{0}(s)\right|^{2} d s & \leqslant C \Delta t+C \sum_{k=0}^{n-1} \int_{t_{k}}^{t_{k+1}} \int_{0}^{T_{0}} \mathbb{E}\left|I\left(r^{h}(s)-r^{h}\left(t_{k}\right)\right)(y)\right|^{2} d y d s \\
& \leqslant C \Delta t+C \Delta t \sum_{k=0}^{n-1} \sup _{t_{k} \leqslant s \leqslant t_{k+1}} \max _{y \in \Gamma \cup \Gamma_{e}} \mathbb{E}\left|r^{h}(s, y)-r^{h}\left(t_{k}, y\right)\right|^{2} .
\end{aligned}
$$


Here we have used Lemma 3.5 and $(3.8)$ to derive the last inequality. Further, it follows from again Lemma 3.5. Assumption 3.1, and (3.8) that for $k=0, \ldots, n-1, s \in\left[t_{k}, t_{k+1}\right]$, $y \in[0, R]$,

$$
\begin{aligned}
& \mathbb{E}\left|r^{h}(s, y)-r^{h}\left(t_{k}, y\right)\right|^{2} \\
& \leqslant 2 \mathbb{E}\left|I^{\prime}\left(r^{h}\left(t_{k}\right)\right)(y)+\alpha\left(t_{k}, I\left(r^{h}\left(t_{k}\right)\right)\right)(y)\right|^{2}(\Delta t)^{2}+2 \sum_{i=1}^{d} \mathbb{E}\left|\sigma_{i}\left(t_{k}, I\left(r^{h}\left(t_{k}\right)\right)\right)(y)\right|^{2} \Delta t \\
& \leqslant C\left(1+\max _{j=1, \ldots, N} \mathbb{E}\left|r^{h}\left(t_{k}, x_{j}\right)\right|^{2}\right) \Delta t .
\end{aligned}
$$

From this and Lemma 3.7, we deduce

$$
\mathcal{I}_{5} \leqslant C \Delta t, \quad i=1, \ldots, d
$$

Similarly, we obtain

$$
\mathcal{I}_{6} \leqslant C \Delta t+C(\Delta x)^{(2 \tau-1) / \tau} R^{1 / \tau}+C \int_{0}^{t} \max _{y \in \Gamma \cup \Gamma_{e}} \mathbb{E}\left|r(s, y)-r^{h}(s, y)\right|^{2} d s .
$$

Consequently, (3.6), (3.7), 3.11)-(3.15) yield

$\max _{x \in \Gamma \cup \Gamma_{e}} \mathbb{E}\left|r(t, x)-r^{h}(t, x)\right|^{2} \leqslant C \Delta t+C(\Delta x)^{(2 \tau-1) / \tau} R^{1 / \tau}+C \int_{0}^{t} \max _{x \in \Gamma \cup \Gamma_{e}} \mathbb{E}\left|r(s, x)-r^{h}(s, x)\right|^{2} d s$.

Finally, applying Gronwall's lemma for the function $t \mapsto \max _{x \in \Gamma \cup \Gamma_{e}} \mathbb{E}\left|r(t, x)-r^{h}(t, x)\right|^{2}$, we complete the proof of the theorem.

\section{Numerical examples}

In this section, we give two numerical illustrations of our collocation method.

Example 4.1 (Vasicek model). The HJMM equation corresponding to Vasicek model is described as

$$
d r(t, x)=\left(\frac{\partial}{\partial x} r(t, x)+\sigma(x) \int_{0}^{x} \sigma(y) d y\right) d t+\sigma(x) d W_{1}(t)
$$

where $\sigma(x)=\sigma e^{-\lambda x}, \sigma, \lambda>0$, and

$$
r(0, x)=r_{0} e^{-\lambda x}+b\left(1-e^{-\lambda x}\right)-\frac{\sigma^{2}}{2 \lambda^{2}}\left(1-e^{-\lambda x}\right)^{2} .
$$

The unique mild to solution $r(t, x)$ to this equation is given by

$$
\begin{aligned}
r(t, x) & =r(0, t+x)+\int_{0}^{t} \sigma(t-s+x) \int_{0}^{t-s+x} \sigma(y) d y d s+\int_{0}^{t} \sigma(t-s+x) d W_{1}(s) \\
& =r(0, t+x)+\frac{\sigma^{2}}{2 \lambda^{2}}\left(2 e^{-\lambda x}\left(1-e^{-\lambda t}\right)-e^{-2 \lambda x}\left(1-e^{-2 \lambda t}\right)\right)+\sigma \int_{0}^{t} e^{-\lambda(t-s+x)} d W_{1}(s) .
\end{aligned}
$$


We examine our collocation method to this model with the following parameters: $T=1$, $\sigma=0.1, b=0.02, \lambda=1.0$, and $r_{0}=0.02$. We use the Wendland kernel $\phi_{4}$ scaled by some positive constant for the performance test. We choose the time grid as a uniform one in $[0,1]$, and as suggested in Remark 3.3 , we define $\Gamma$ by the uniform spatial grid points on $[R /(N+1), R-R /(N+1)]$ where $R=(1 / 5) N^{(1-1 /(2 \tau-2))}$, while the set of evaluation points $\Gamma_{e}=\left\{\xi_{1}, \ldots, \xi_{100}\right\}$ by a Sobol' sequence on $[R / 4,3 R / 4]$.

It should be remarked that this model satisfies Assumption 3.1 with $w(x)=e^{\lambda x}$ and $\Psi(x)=\sigma\left(1+\lambda+\lambda^{2}\right) e^{-\lambda x}$. To check the validity of Assumption 3.2 (ii), we plot

$$
\iota(N)=\max _{m=0,1,2} \max _{x \in \Gamma \cup \Gamma_{e}} \#\left\{j:\left|\frac{d^{m}}{d x^{m}} Q_{j}(x)\right|>\frac{25}{N}\right\}
$$

in Figure 4.1. We can see that $\iota(N)<25 \sqrt{R}$ for all $N \leqslant 1000$. Thus, Assumption 3.2 (ii)

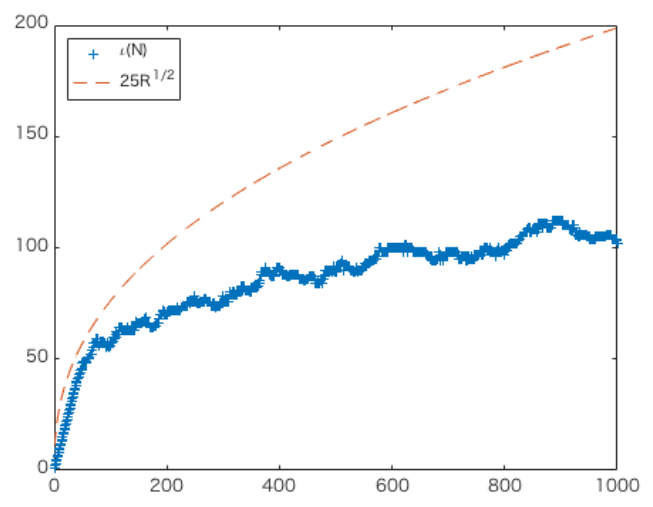

Figure 4.1: Plotting $\iota(N)$ and $25 \sqrt{R}$ for $N=1,2, \ldots, 1000$.

seems to be satisfied with $c_{1}=25$ and $c_{2}=25$ for the sequence of the tuning parameters defined by $N$ from 1 at least to 1000 .

To compare an averaged performance, we compute the root mean squared errors averaged over 10000 samples, defined by

$$
\operatorname{RMSE}:=\sqrt{\frac{1}{10000 \times 100(n+1)} \sum_{i=0}^{n} \sum_{j=1}^{100} \sum_{\ell=1}^{10000}\left|r_{\ell}\left(t_{i}, \xi_{j}\right)-r_{\ell}^{h}\left(t_{i}, \xi_{j}\right)\right|^{2}},
$$

for several values of $N$ and $n$. Here, $r_{\ell}$ and $r_{\ell}^{h}$ are the exact solution and approximate solution at $\ell$-th trial, respectively.

Table 4.1 shows that the resulting RMSE's are sufficiently small for all pairs $(N, n)$ although its decrease is nonmonotonic. This illustrates the convergence result given in Theorem 3.4.

Example 4.2 (Pricing of caplets). Here, we apply our approximation methods to the pricing of the caplet. To this end, we consider the forward 6 months LIBOR prevailing 


\begin{tabular}{cccc}
\hline$N$ & $R$ & $n$ & RMSE \\
\hline $2^{6}$ & 6.4000 & $2^{4}$ & 0.0180 \\
& & $2^{6}$ & 0.0343 \\
& & $2^{8}$ & 0.0683 \\
\hline $2^{7}$ & 11.4035 & $2^{4}$ & 0.0037 \\
& & $2^{6}$ & 0.0074 \\
& & $2^{8}$ & 0.0146 \\
\hline $2^{8}$ & 20.3187 & $2^{4}$ & $2.9578 \mathrm{e}-04$ \\
& & $2^{6}$ & $5.9918 \mathrm{e}-04$ \\
& & $2^{8}$ & 0.0012 \\
\hline
\end{tabular}

Table 4.1: The resulting root mean squared errors for several pairs $(N, n)$.

at time $t$ over the future period [T,T+0.5], defined by

$$
1+0.5 L(t, T)=\frac{P(t, T)}{P(t, T+0.5)},
$$

or

$$
L(t, T)=\frac{1}{0.5}\left(\exp \left(\int_{T}^{T+0.5} f(t, s) d s\right)-1\right)=\frac{1}{0.5}\left(\exp \left(\int_{T-t}^{T-t+0.5} r(t, x) d x\right)-1\right),
$$

where $P(t, T)$ and $f(t, s)$ are the price of the discounted bond and the forward rate, respectively, given in Section 2 . Then the caplet price with $T=10$ is given by

$$
0.5 \mathbb{E}\left[e^{-\int_{0}^{T+0.5} r(s, 0) d s} \max (L(T, T)-\kappa, 0)\right] .
$$

As the volatility functional $\sigma_{i}$ 's, we examine the 5 year yield-dependent model

$$
\sigma_{1}(t, \phi)(x)=\theta_{1} e^{-\theta_{2} x} \max \left(0, \min \left(\frac{1}{5} \int_{0}^{5} \phi(y) d y, 1\right)\right),
$$

which is addressed in [8] in a slightly different form. As in the previous example, Assumption 3.1 is satisfied with $w(x)=e^{\theta_{2} x}$ and $\Psi(x)=\theta_{1}\left(1+\theta_{2}+\theta_{2}^{2}\right) e^{-\theta_{2} x}$.

We use the Wendland kernel $\phi_{4}$ scaled by some positive constant. The time and spatial grids are set to be $t_{i}=i 10^{-4}, i=0,1, \ldots, 10^{5}$, and $x_{j}=j / 5, j=1, \ldots, 50$, respectively. To confirm Assumption 3.2 (ii) numerically, we consider the uniform spatial grid points on $[R / N, R]$ where $R=(2 / 5) N^{\log 25 / \log 50}$ so that $R=10$ for $N=50$.

We can see that $\iota(N)<25 \sqrt{R}$ for all $N \leqslant 1000$ in Figure 4.2. Thus, Assumption 3.2 (ii) seems to be satisfied with $c_{1}=12$ and $c_{2}=25$ for the sequence of the tuning parameters defined by $N$ from 1 at least to 1000 .

Let $r^{h}$ be the approximate solution, described in Section 3 , of the HJMM equation with the yield-dependent volatility model above. Then, (4.1) is approximated by

$$
0.5 \mathbb{E}\left[e^{-10^{-5} \sum_{i=0}^{k} r^{h}\left(t_{i}, \xi_{1}\right)} \max \left(L^{h}(T, T)-\kappa, 0\right)\right],
$$




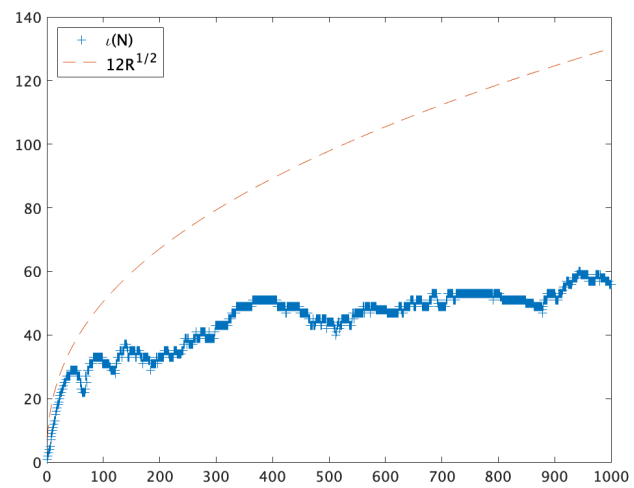

Figure 4.2: Plotting $\iota(N)$ and $12 \sqrt{R}$ for $N=1,2, \ldots, 1000$.

with

$$
L^{h}(T, T)=\frac{1}{0.5}\left(\exp \left(r^{h}\left(T, \xi_{1}\right) / 10+r^{h}\left(T, \xi_{2}\right) / 5+7 r^{h}\left(T, \xi_{3}\right) / 40+r^{h}\left(T, \xi_{4}\right) / 40\right)-1\right),
$$

where $\Gamma_{e}=\left\{\xi_{j}\right\}$ is the set of the evaluation points given by $\xi_{j}=(j-1) / 5, j=1, \ldots, 51$ and we have used the approximation

$$
\begin{aligned}
\int_{0}^{0.5} r^{h}(T, x) d x \approx & \frac{1}{2}\left(r^{h}\left(T, \xi_{1}\right)+r^{h}\left(T, \xi_{2}\right)\right) \times 0.2+\frac{1}{2}\left(r^{h}\left(T, \xi_{2}\right)+r^{h}\left(T, \xi_{3}\right)\right) \times 0.2 \\
& +\frac{1}{2}\left(r^{h}\left(T, \xi_{3}\right)+\frac{1}{2}\left(r^{h}\left(T, \xi_{3}\right)+r^{h}\left(T, \xi_{4}\right)\right)\right) \times 0.1 .
\end{aligned}
$$

Figure 4.3 plots the approximated price 4.2 with $N=50$ for $\theta_{1} \in\{0.05,0.1, \ldots, 2.5\}$ and $\theta_{2} \in\{0.05, \ldots, 1.5\}$. We can confirm a regular behavior of our approximation with respect to the changes of the parameters $\theta_{1}$ and $\theta_{2}$.

\section{Acknowledgements}

This study is supported by JSPS KAKENHI Grant Number JP17K05359. The authors are thankful to Kanji Kurihara and Hideki Noda for helping us perform the numerical experiments in Section 4 .

\section{References}

[1] M. Barski and J. Zabczyk. Heath-Jarrow-Morton-Musiela equation with Lévy perturbation. J. Differential Equations, 253:2657-2697, 2012.

[2] A. Barth. A finite element method for martingale-driven stochastic partial differential equations. Commun. Stochast. Anal., 4:355-375, 2010. 


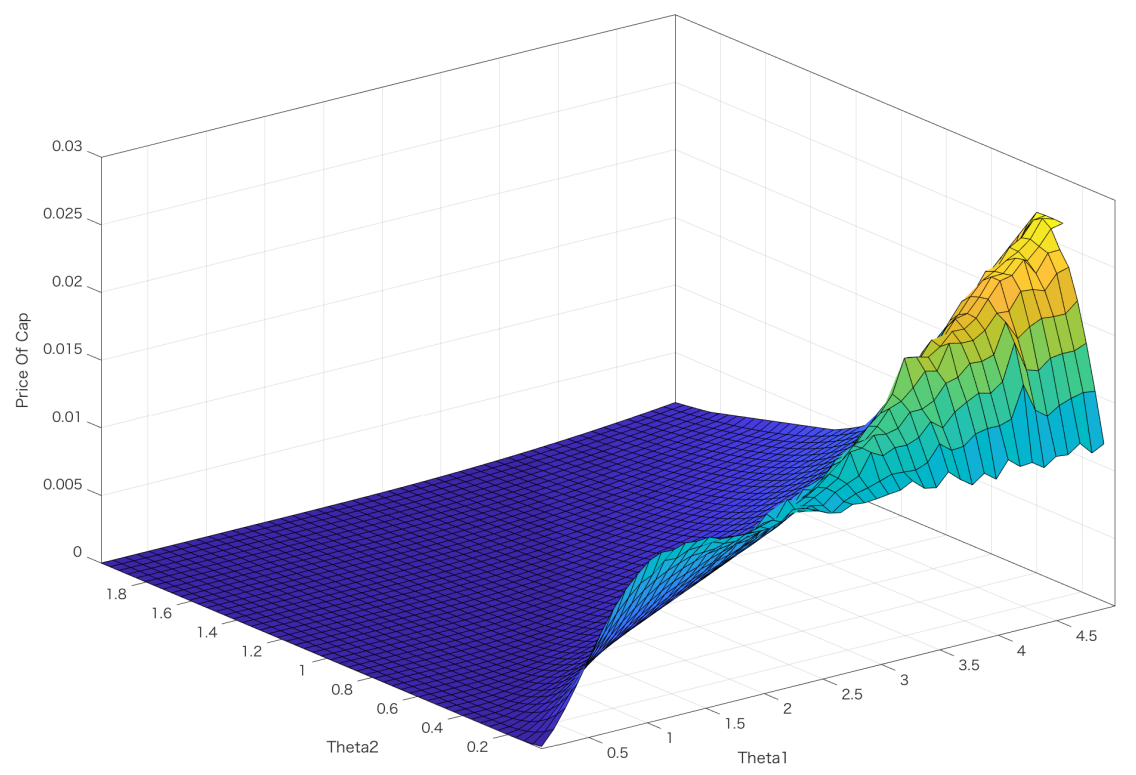

Figure 4.3: Plotting the approximated prices in the cases of $T=10, \kappa=0.04$, and $\left(\theta_{1}, \theta_{2}\right) \in\{0.05,0.1, \ldots, 2.5\} \times\{0.05, \ldots, 1.5\}$. 
[3] T. Björk. Arbitrage theory in continuous time. Oxford University Press, Oxford, 2nd edition, 2004.

[4] A. Chernih, I. H. Sloan, and R. S. Womersley. Wendland functions with increasing smoothness converge to a Gaussian. Adv. Comput. Math., 40:185-200, 2014.

[5] I. Cialenco, G. E. Fasshauer, and Q. Ye. Approximation of stochastic partial differential equations by a kernel-based collocation methods. Int. J. Comput. Math., 89:2543-2561, 2012.

[6] G. Da Prato and J. Zabczyk. Stochastic equations in infinite dimensions. Cambridge University Press, Cambridge, 2014.

[7] P. Dörsek and J. Teichmann. Efficient simulation and calibration of general HJM models by splitting schemes. SIAM J. Finan. Math., 4:575-598, 2013.

[8] D. Filipović. Consistency problems for Heath-Jarrow-Morton interest rate models. Springer, New York, 2001.

[9] B. Goldys and M. Musiela. Infinite dimensional diffusions, Kolmogorov equations and interest rate models. In E. Jouini, J. Cvitanić, and M. Musiela, editors, Option pricing, interest rates and risk management, pages 314-335. Cambridge University Press, Cambridge, 2001.

[10] D. Heath, R. Jarrow, and A. Morton. Bond pricing and the term structure of interest rates: A new methodology for contingent claim valuation. Econometrica, 60:77-105, 1992.

[11] Y. C. Hon, R. Schaback, and M. Zhong. The meshless kernel-based method of line for parabolic equations. Comput. Math. Appl., 68:2057-2067, 2014.

[12] E. J. Kansa. Application of Hardy's multiquadric interpolation to hydrodynamics. In Proc. 1986 Simul. Conf., pages 111-117, 1986.

[13] E. J. Kansa. Multiquadricsa scattered data approximation scheme with application to computational fluid-dynamicsI. Computers Math. Applic., 19:127-145, 1990.

[14] E. J. Kansa. Multiquadricsa scattered data approximation scheme with application to computational fluid-dynamicsII. Computers Math. Applic., 19:147-161, 1990.

[15] S. Kusuoka. Term structure and SPDE. Adv. Math. Econ., 2:67-85, 2000.

[16] M. Musiela. Stochastic PDEs and term structure models. Journées internationales de finance, IGR-AFFI, La Baule, 1993.

[17] M. Musiela and M. Rutkowski. Martingale methods in financial modelling. Springer, Berlin, 2nd edition, 2007.

[18] Y. Nakano. Convergent kernel-based methods for nonlinear parabolic equations. arXiv:1803.09446[Math.NA]. 
[19] Y. Nakano. Convergence of meshfree collocation methods for fully nonlinear parabolic equations. Numer. Math., 136:703-723, 2017.

[20] Y. Nakano. Kernel-based collocation methods for Zakai equations. Stoch. Partial Differ. Equ. Anal. Comput., 7:476-494, 2019. (A corrected version is available at https://arxiv.org/abs/1710.09090).

[21] R. Schaback. Unsymmetric meshless methods for operator equations. Numer. Math., 114:629-651, 2010.

[22] S. E. Shreve. Stochastic calculus for finance II. Springer, New York, 2004.

[23] H. Wendland. Scattered data approximation. Cambridge University Press, Cambridge, 2010. 